\title{
A Kleene-Schützenberger Theorem for Weighted Timed Automata
}

\author{
Manfred Droste and Karin Quaas \\ Institut für Informatik, Universität Leipzig \\ 04009 Leipzig, Germany \\ \{droste, quaas\}@informatik. uni-leipzig.de
}

\begin{abstract}
During the last years, weighted timed automata (WTA) have received much interest in the real-time community. Weighted timed automata form an extension of timed automata and allow us to assign weights (costs) to both locations and edges. This model, introduced by Alur et al. (2001) and Behrmann et al. (2001), permits the treatment of continuous consumption of resources and has led to much research on scheduling problems, optimal reachability and model checking. Also, several authors have derived Kleene-type characterizations of (unweighted) timed automata and their accepted timed languages. The goal of this paper is to provide a characterization of the possible behaviours of WTA by rational power series. We define WTA with weights taken in an arbitrary semiring, resulting in a model that subsumes several WTA concepts of the literature. For our main result, we combine the methods of Schützenberger, a recent approach for a Kleene-type theorem for unweighted timed automata by Bouyer and Petit as well as new techniques. Our main result also implies Kleene-type theorems for several subclasses of WTA investigated before, e.g., for weighted finite automata, timed automata and timed automata with stopwatch observers.
\end{abstract}

\section{Introduction}

Since its introduction in 1994 by Alur and Dill [2], timed automata have been a thoroughly investigated model for the specification and analysis of real-time systems. In the literature, not only a variety of interesting theoretical results for timed automata and timed languages have been established (see [4] for a survey), but there has also much practical work been done such as the development of symbolic data structures and efficient algorithms, leading to model-checking tools like Kronos, UPPAAL and HyTECH [17, 25], 22, successfully used for solving industrially relevant problems, e.g. 27], 23].

Weighted timed automata have been of much interest in the real-time community during the last years. The model has been introduced independently by Alur et al. 3] and Behrmann et al. 8] and allows us to assign weights to both the locations and edges of the underlying timed automaton. The weight of an edge gives the actual cost for executing it, whereas the weight of a location gives the cost for staying in this location per time unit. The weight for reaching a certain 
location $s$ (or, analogously, the weight for accepting a certain timed word) is computed by taking the minimum over the running weights of all runs ending in $s$. The running weight of a run is the sum of the costs of all participating transitions of the run. In this way, WTA have been used to model continuous consumption of resources, allowing applications in operations research, in particular optimal scheduling and planning [20, 28]. Consequently, a number of decision problems have been investigated, most of them concerning the reachability problem under some optimization aspect [8], 3], 11], model-checking [18], [12, [14, and weighted timed games [1].

The goal of this paper is to provide a characterization of the possible behaviours of WTA in terms of rational power series, i.e., power series constructed by the standard rational operations addition, Cauchy product and Kleene star iteration. Moreover, we give a more general definition of WTA which includes all the various definitions given in the literature so far and which gives rise to some interesting variants.

We define WTA over a semiring in the same manner as it is done for classical weighted finite automata [29], 24], [10]. In this way, we are not bound to a fixed set of weights, nor are we restricted to use the operations of addition and infimum for computing the weight of a word. Secondly, we do not restrict the cost functions for the locations to be linear (as previously done in [3], [8]). Instead, we consider WTA with respect to an arbitrary family of functions $\mathcal{F}$ mapping positive reals to elements in the semiring. The cost for staying in a location $s$ is defined by a cost function $C_{s} \in \mathcal{F}$. By introducing the notion of a family of cost functions $\mathcal{F}$ and the semiring, we hope to obtain a flexible model of WTA.

For our characterization of the behaviours of WTA by rational power series, we establish a Kleene-Schützenberger theorem for WTA. Schützenberger's theorem is the analogue to the famous Kleene theorem for the class of weighted finite automata: the set of recognizable power series, i.e., the set of power series that constitute the behaviour of a weighted automaton, is precisely the set of rational power series [30]. As is well-known, rational expressions can be used to specify properties of systems. Recently, there have also been several approaches to give a Kleene-type theorem for timed languages [5], 7], 6], 15], of which we choose the latest approach of Bouyer and Petit in 2001 [16] because of its simplicity and elegance. According to their result, the set of regular timed languages, as defined by Alur and Dill [2, coincides with the set of rational timed languages, defined over the standard rational operations $+, \cdot,{ }^{*}$ and an additional projection operation. For the proof of our main result, we combine the methods of Bouyer and Petit, Schützenberger and new techniques.

We define the semantics of WTA based on the notion of clock words as introduced by Bouyer and Petit 16. Clock words, as opposed to the well-known timed words [2], bear more information concerning the actual values of clock variables than timed words, and thus enable the authors to define a concatenation operation in a natural way. Consequently, all the definitions and constructions for the Kleene theorem are given with respect to clock words. However, it can be shown that clock words can easily be mapped to timed words using a projection 
function; thus the Kleene theorem for clock words can be extended to timed words. To bring weights into play, we introduce the notion of clock series. Clock series are a particular kind of power series which map clock words to elements in a semiring. We define addition, Cauchy product and Kleene star iteration on the set of clock series to give a formal definition for the set of rational clock series. The main objective of this work is to show that this set is equal to the set of recognizable clock series, which make up the behaviour of the class of WTA. We establish this in two steps. First, the crucial part for showing that any rational clock series is a recognizable clock series is to prove that WTA are closed under the three operations mentioned above. In our proof, for dealing with the weights assigned to locations, we need to give new methods for normalizing the automata. The proof for the other direction, i.e., any recognizable clock series is rational, is based on the solution of equations [16], [10. Finally, we show how we can extend the theorem in such a way that it can be applied to timed semantics as well.

\section{Preliminaries}

In the following, we use $\mathbb{R}_{\geq 0}$ and $\mathbb{N}$ to denote the positive reals and natural numbers, respectively. Furthermore, we write $\mathbb{R}_{\geq 0}^{1+n}$ for $\mathbb{R}_{\geq 0} \times \mathbb{R}_{\geq 0}^{n}$.

Clock Constraints and Clock Valuations. Let $X$ be a finite set of variables, called clock variables. We define clock constraints $\phi$ over $X$ to be conjunctions of formulas of the form $x \sim k$, where $k \in \mathbb{N}, x \in X$, and $\sim \in\{<, \leq,>, \geq\}$. Let $\Phi(X)$ be the set of all clock constraints $\phi$ over $X$. A clock valuation $\nu: X \rightarrow \mathbb{R}_{\geq 0}$ is a function that assigns a value to each clock variable. A clock valuation $\nu$ satisfies a clock constraint $\phi$, written $\nu \models \phi$, if $\phi$ evaluates to true according to the values given by $\nu$. Given $\delta \in \mathbb{R}_{\geq 0}$, we let $\nu+\delta$ be the clock valuation such that $(\nu+\delta)(x)=\nu(x)+\delta$ for each $x \in X$. For $\lambda \subseteq X$, we define $\nu[\lambda:=0]$ as the clock valuation that assigns 0 to each $x \in \lambda$, and agrees with $\nu$ over the remaining clock variables $x \in X \backslash \lambda$.

Timed and Clock Words. Let $\Sigma$ be a finite alphabet and $n \in \mathbb{N}$. A timed word is a finite sequence $w=\left(\sigma_{1}, t_{1}\right)\left(\sigma_{2}, t_{2}\right) \ldots\left(\sigma_{k}, t_{k}\right) \in\left(\Sigma \times \mathbb{R}_{\geq 0}\right)^{*}$, where the sequence $t_{1} t_{2} \ldots t_{k}$ is non-decreasing. Intuitively, $t_{i}$ gives the time of occurrence of $\sigma_{i}$. An $n$-clock word is a finite sequence $w=\left(t_{0}, \nu_{0}\right)\left(\sigma_{1}, t_{1}, \nu_{1}\right) \ldots\left(\sigma_{k}, t_{k}, \nu_{k}\right)$ from the infinite set $\left(\mathbb{R}_{>0}^{1+n}\right)\left(\Sigma \times \mathbb{R}_{>0}^{1+n}\right)^{*}$, where $\left(\sigma_{1}, t_{1}\right) \ldots\left(\sigma_{k}, t_{k}\right)$ is a timed word, and $\nu_{i}$ gives the values of the clock variables just after the computation of $\sigma_{i}$. The pair $\left(t_{0}, \nu_{0}\right)$ corresponds to the starting condition and is considered to be an empty $n$-clock word. The set of empty $n$-clock words is denoted by $\Gamma_{n}\left(=\mathbb{R}_{\geq 0}^{1+n}\right)$. We define $\left(\mathbb{R}_{\geq 0}^{1+n}\right)\left(\Sigma \times \mathbb{R}_{\geq 0}^{1+n}\right)^{+}$by $\left(\mathbb{R}_{\geq 0}^{1+n}\right)\left(\Sigma \times \mathbb{R}_{\geq 0}^{1+n}\right)^{*} \backslash \Gamma_{n}$. Let $w=\left(t_{0}, \nu_{0}\right)\left(\sigma_{1}, t_{1}, \nu_{1}\right) \ldots\left(\sigma_{k}, t_{k}, \nu_{k}\right)$ and $w^{\prime}=\left(t_{0}^{\prime}, \nu_{0}^{\prime}\right)\left(\sigma_{1}^{\prime}, t_{1}^{\prime}, \nu_{1}^{\prime}\right) \ldots\left(\sigma_{l}^{\prime}, t_{l}^{\prime}, \nu_{l}^{\prime}\right)$ be two $n$-clock words. We say that $w$ is compatible with $w^{\prime}$ if $\left(t_{k}, \nu_{k}\right)=\left(t_{0}^{\prime}, \nu_{0}^{\prime}\right)$. In this case, we define the concatenation $w \cdot w^{\prime}$ of $w$ and $w^{\prime}$ to be the $n$-clock word $\left(t_{0}, \nu_{0}\right)\left(\sigma_{1}, t_{1}, \nu_{1}\right) \ldots\left(\sigma_{k}, t_{k}, \nu_{k}\right)\left(\sigma_{1}^{\prime}, t_{1}^{\prime}, \nu_{1}^{\prime}\right) \ldots\left(\sigma_{l}^{\prime}, t_{l}^{\prime}, \nu_{l}^{\prime}\right)$. By $|w|$ we mean the length of an $n$-clock word $w$. 
Semirings and Formal Power Series. A semiring is a tuple $\mathcal{K}=(K, \oplus, \odot, 0,1)$ such that $(K, \oplus, 0)$ is a commutative monoid, $(K, \odot, 1)$ is a monoid, $\odot$ is both left- and right-distributive over $\oplus$, and $0 \odot x=x \odot 0=0$ for any $x \in K$. As examples consider the semiring $(\mathbb{N},+, \cdot, 0,1)$ of natural numbers with the usual addition and multiplication, the Boolean semiring $(\{0,1\}, \vee, \wedge, 0,1)$, and the tropical semiring $\left(\mathbb{R}_{\geq 0} \cup\{\infty\}\right.$, min, $\left.+, \infty, 0\right)$. Let $A$ be an arbitrary set and $\mathcal{K}$ a semiring. A function $S: A \rightarrow K$ is called a formal power series (fps) over $\mathcal{K}$. For historical reasons, the image of an element $w \in A$ is denoted by $(S, w)$. We write $\mathcal{K}\langle\langle A\rangle\rangle$ to mean the set of all fps $S: A \rightarrow K$.

\section{Weighted Timed Automata}

Weighted Timed Automata. Let $\mathcal{K}$ be a semiring, $\Sigma$ be a finite alphabet, and $X$ be a finite set of clock variables. We consider timed automata $\mathcal{A}$ augmented with cost functions that assign elements from $K$, so-called weights (or costs), to both the edges and the locations of $\mathcal{A}$. The weight for staying in a location depends on the amount of time we spend in this location; thus, we define a cost function from $\mathbb{R}_{\geq 0}$ to $K$ for each location. Let $\mathcal{F}$ be any family of functions from $\mathbb{R}_{\geq 0}$ to $K$. A weighted timed automaton (WTA) over $\mathcal{K}, \Sigma, X$ and $\mathcal{F}$ is a tuple $\mathcal{A}=\left(S, S_{0}, S_{f}, E, C\right)$, where

$-S$ is a finite set of locations (states)

$-S_{0} \subseteq S$ is a set of initial locations

$-S_{f} \subseteq S$ is a set of final locations

- $E \subseteq S \times S \times \Sigma \times \Phi(X) \times 2^{X}$ is a finite set of edges. An edge $\left(s, s^{\prime}, \sigma, \phi, \lambda\right)$ allows a jump from location $s$ to location $s^{\prime}$ if $\sigma$ is read, provided that the current values of the clock variables in location $s$ satisfy the clock constraint $\phi$. After the edge has been executed, all clock variables in $\lambda$ are reset to zero, whereas the values of all other clock variables remain unchanged.

$-C=\left\{C_{E}\right\} \cup\left\{C_{s}: s \in S\right\}$, where $C_{E}: E \rightarrow K$, and $C_{s} \in \mathcal{F}$ for any $s \in S$.

Let $\mathcal{A}=\left(S, S_{0}, S_{f}, E, C\right)$ be a WTA. The timed semantics of $\mathcal{A}$ is given by an infinite state transition system that corresponds to a weighted extension of the original semantics of timed automata defined by Alur and Dill [2]. However, in the following we will give an additional semantics, called clock semantics. This model is based on the notion of clock words rather than timed words, and allows for a natural definition of concatenation [16].

Timed Semantics. Let $\mathcal{S}_{\mathcal{A}}^{T}$ be a state-transition-system with states of the form $(s, \nu)$, where $s \in S$ and $\nu$ is a clock valuation. We define timed transitions to be transitions of the form $(s, \nu) \stackrel{\delta / c}{\longrightarrow} T(s, \nu+\delta)$ where $c=C_{s}(\delta)$. A discrete transition is of the form $(s, \nu) \stackrel{\sigma / c}{\longrightarrow} D\left(s^{\prime}, \nu^{\prime}\right)$ such that there is an edge $e=\left(s, s^{\prime}, \sigma, \phi, \lambda\right)$ in $E$ where $C_{E}(e)=c, \nu=\phi$, and $\nu^{\prime}=\nu[\lambda:=0]$. A timed run $r^{T}$ of $\mathcal{A}$ is a finite alternating sequence of timed and discrete transitions in $\mathcal{S}_{\mathcal{A}}^{T}$

$$
r^{T}=\left(\left(s_{0}, \nu_{0}\right) \stackrel{\delta_{1} / c_{1}}{\longrightarrow} T\left(s_{1}, \nu_{1}\right) \stackrel{\sigma_{1} / c_{1}^{\prime}}{\longrightarrow} D\left(s_{1}^{\prime}, \nu_{1}^{\prime}\right) \stackrel{\delta_{2} / c_{2}}{\longrightarrow} T \ldots \stackrel{\sigma_{k} / c_{k}^{\prime}}{\longrightarrow} D\left(s_{k}^{\prime}, \nu_{k}^{\prime}\right)\right)
$$


where $\nu_{0}=0^{|X|}$. With $r^{T}$, the timed word $w=\left(\sigma_{1}, t_{1}\right)\left(\sigma_{2}, t_{2}\right) \ldots\left(\sigma_{k}, t_{k}\right)$, such that $t_{j}=\sum_{i=1}^{j} \delta_{j}$ for each $1 \leq j \leq k$, is associated.

Clock Semantics. The clock semantics is very similar to the timed semantics and is given in terms of $|X|$-clock words. Consider the state-transition-system $\mathcal{S}_{\mathcal{A}}^{C}$, whose states are of the form $(s, t, \nu)$, where $s$ is a location, $t \in \mathbb{R}_{\geq 0}$, and $\nu$ is a clock valuation. The transition relation over the set of states in $\mathcal{S}_{\mathcal{A}}^{\bar{C}}$ is defined in the same manner as the transition relation in $\mathcal{S}_{\mathcal{A}}^{T}$. A clock run $r^{C}$ of $\mathcal{A}$ is a finite alternating sequence of timed and discrete transitions

$r^{C}=\left(\left(s_{0}, t_{0}, \nu_{0}\right) \stackrel{\delta_{1} / c_{1}}{\longrightarrow} T\left(s_{1}, t_{1}, \nu_{1}\right) \stackrel{\sigma_{1} / c_{1}^{\prime}}{\longrightarrow} D\left(s_{1}^{\prime}, t_{1}^{\prime}, \nu_{1}^{\prime}\right) \stackrel{\delta_{2} / c_{2}}{\longrightarrow} T \ldots \stackrel{\sigma_{k} / c_{k}^{\prime}}{\longrightarrow} D\left(s_{k}^{\prime}, t_{k}^{\prime}, \nu_{k}^{\prime}\right)\right)$

where $t_{1}=t_{0}+\delta_{1}, t_{i}^{\prime}=t_{i}$ for any $1 \leq i \leq k$, and $t_{i}=t_{i-1}^{\prime}+\delta_{i}$ for any $2 \leq i \leq k$. Note that in contrast to the timed semantics, $\nu_{0}$ can be arbitrary. The label of a canonical clock run is the $|X|$-clock word $w=\left(t_{0}, \nu_{0}\right)\left(\sigma_{1}, t_{1}^{\prime}, \nu_{1}^{\prime}\right) \ldots\left(\sigma_{k}, t_{k}^{\prime}, \nu_{k}^{\prime}\right)$.

Behaviour of $\mathcal{A}$. Let $r$ be a (timed or clock) run as above. We define the running weight $r w t(r)$ of $r$ to be $\operatorname{rwt}(r)=\prod_{i=1}^{k} c_{i} \odot c_{i}^{\prime}$. The running weight of the empty clock run $\left(t_{0}, \nu_{0}\right)$ with label $\left(t_{0}, \nu_{0}\right) \in \Gamma_{|X|}$ is defined to be 1 . We say that $r$ is initialized if $s_{0} \in S_{0}$. It is accepting if $s_{k}^{\prime} \in S_{f}$. If $r$ is both initialized and accepting it is called successful. The timed behaviour of the WTA $\mathcal{A}$ is the fps $\|\mathcal{A}\|^{T}:\left(\Sigma \times \mathbb{R}_{\geq 0}\right)^{*} \rightarrow K$ defined by

$$
\left(\|\mathcal{A}\|^{T}, w\right)=\sum\{r w t(r): r \text { is a successful timed run of } \mathcal{A} \text { on } w\}
$$

Similarly, we define the clock behavior of $\mathcal{A}$ to be the fps $\|\mathcal{A}\|^{C}:\left(\mathbb{R}_{\geq 0}^{1+|X|}\right)\left(\Sigma \times \mathbb{R}_{\geq 0}^{1+|X|}\right)^{*} \rightarrow K$ given by

$$
\left(\|\mathcal{A}\|^{C}, w\right)=\sum\{r w t(r): r \text { is a successful clock run of } \mathcal{A} \text { on } w\}
$$

In the remainder of the paper, we will use the clock semantics for defining the notions of recognizability and rationality. In the last section, we show that these notions can easily be adapted to the timed semantics.

\section{Relation to other Automata Models}

Here we show that our model of WTA subsumes a number of more particular concepts of timed automata, as well as weighted (untimed) automata, which have been investigated intensively in the literature. In particular, by restricting $\mathcal{K}$ and $\mathcal{F}$, we obtain timed automata and weighted automata. This implies that our main theorem in Sect. 5 also applies to these automata classes.

Timed Automata. The classical (unweighted) timed automaton defined by Alur and Dill [2] can be obtained as follows. Let $\mathcal{K}$ be the Boolean semiring $(\{0,1\}, \vee, \wedge, 0,1), \mathcal{F}$ be the family of constant functions 1 , i.e., $C_{s}(\delta)=1$ for any $s \in S, \delta \in \mathbb{R}_{\geq 0}$, and $C_{E}(e)=1$ for any $e \in E$. Thus, Theorem 8 of Bouyer and Petit [16] is implied by our main theorem in Sect. 5 . 
Other Weighted Timed Automata Models. Weighted timed automata have been introduced independently by Alur et al. [3] and Behrmann et al. 8]. Both consider timed automata $\mathcal{A}$ augmented with a cost function that assigns a natural number to locations and edges of $\mathcal{A}$. In doing so, the increase of the cost variable is restricted to be linear. The cost of reaching a location $s$ is computed by taking the minimum of the costs of any run ending in $s$, where the cost of a run $r$ is the sum of the costs of all participating transitions in $r$. We can easily model this using the tropical semiring $\left(\mathbb{R}_{\geq 0} \cup\{\infty\}, \min ,+, \infty, 0\right)$, and restricting $\mathcal{F}$ to the class of linear functions.

Recently, the weighted timed automaton model has been generalized by allowing more than one cost variable. Larsen and Rasmussen introduced dual-priced timed automata [26]. The dual-priced timed automaton model can be modeled by defining a new "tropical" semiring with the underlying set $\left(\mathbb{R}_{\geq 0} \cup\{\infty\}\right) \times$ $\left(\mathbb{R}_{\geq 0} \cup\{\infty\}\right)$, and modifying the definitions of min and + in a suitable way, e.g. coordinate-wise. Similarly, we can extend to multi-priced models 13 .

Timed Automata with Stopwatch Observers. A stopwatch is a clock variable that can be stopped and turned on again [21. In other words, the rate of change of the stopwatch variable is either 0 or 1 . A timed automaton augmented with a stopwatch variable that can neither be tested in a clock constraint nor be reset is called a timed automaton with a stopwatch observer. We use a WTA to model such an automaton by restricting $\mathcal{F}$ to be the constant functions 0 and 1. The edges shall not cost anything: $C_{E}(e)=1$ for any $e \in E$.

Weighted Finite Automata. A weighted finite automaton $\mathcal{A}$ over a semiring $\mathcal{K}=(K, \oplus, \odot, 0,1)$ is a finite automaton whose transitions are assigned costs taken from the semiring. The behavior of $\mathcal{A}$ is defined using the semiring operations $\oplus$ and $\odot$ in the same manner as it is done for WTA in Sect. 3. By restricting the family of functions $\mathcal{F}$ to the constant function 1 , we yield a model which does not add any costs while staying in a location. In this way, we yield a classical weighted finite automaton. This implies that the Schützenberger theorem [30] is a special case of our main theorem in Sect. 5 .

\section{Clock Series}

To describe the behaviour of a WTA $\mathcal{A}$ over $\mathcal{K}, \Sigma, X$ and $\mathcal{F}$, we want to use $\mathcal{F}$ rational clock series. In this section, we give a general definition of clock series, some basic properties of clock series, and the definition of rationality. Finally, we will give the main theorem of the paper. For the remainder of the paper, we fix a semiring $\mathcal{K}$, a finite alphabet $\Sigma$, a set of clock variables $X=\left\{x_{1}, \ldots, x_{n}\right\}$, and a family $\mathcal{F}$ of functions from $\mathbb{R}_{\geq 0}$ to $K$. If not otherwise specified, by writing $\mathcal{A}$ we mean a WTA $\mathcal{A}$ over $\mathcal{K}, \Sigma, X$ and $\mathcal{F}$.

Clock Series. A function $S:\left(\mathbb{R}_{\geq 0}^{1+n}\right)\left(\Sigma \times \mathbb{R}_{\geq 0}^{1+n}\right)^{*} \rightarrow K$ is called an $n$-clock series. We denote the set of all $n$-clock series by $\mathcal{K}_{n}^{-}\left\langle\left\langle\Sigma, \mathbb{R}_{\geq 0}\right\rangle\right\rangle$. On the set $\mathcal{K}_{n}\left\langle\left\langle\Sigma, \mathbb{R}_{\geq 0}\right\rangle\right\rangle$, 
we define the sum $S+T$ pointwise, i.e., $(S+T, w)=(S, w) \oplus(T, w)$. The Cauchy product $S \cdot T$ is defined by

$$
(S \cdot T, w)=\sum_{u \cdot v=w}(S, u) \odot(T, v)
$$

Furthermore, we define the clock series $\mathbb{1}$ by $(\mathbb{1}, w)=1$ if $w \in \Gamma_{n},(\mathbb{1}, w)=0$ otherwise, and the clock series $\mathbb{0}$ by $(\mathbb{O}, w)=0$ for each $w \in\left(\mathbb{R}_{\geq 0}^{1+n}\right)\left(\Sigma \times \mathbb{R}_{\geq 0}^{1+n}\right)$. The following lemma is the clock series version of the well-known fact that the set of fps over the free monoid together with sum and Cauchy product is a semiring.

Lemma 1. The structure $\left(\mathcal{K}_{n}\left\langle\left\langle\Sigma, \mathbb{R}_{\geq 0}\right\rangle\right\rangle,+, \cdot, \mathbb{0}, \mathbb{1}\right)$ is a semiring.

For a clock series $S$, let $S^{0}=\mathbb{1}$ and, inductively, $S^{k}=S \cdot S^{k-1}$ be the $k$-th power of $S$ for $k \geq 1$. The clock series $S \in \mathcal{K}_{n}\left\langle\left\langle\Sigma, \mathbb{R}_{\geq 0}\right\rangle\right\rangle$ is called proper, if $\left(S, w_{0}\right)=0$ for any $w_{0} \in \Gamma_{n}$. For proper clock series $S$, we define the Kleene star iteration $S^{*}$ by

$$
\left(S^{*}, w\right)=\sum_{k \geq 0}\left(S^{k}, w\right)
$$

Notice that from $\left(S, w_{0}\right)=0$ for $w_{0} \in \Gamma_{n}$, it follows that $\left(S^{k}, w\right)=0$ for any $k>|w|$. This implies that the sum given above is finite and hence exists in $\mathcal{K}$.

Lemma 2. Let $S \in \mathcal{K}_{n}\left\langle\left\langle\Sigma, \mathbb{R}_{\geq 0}\right\rangle\right\rangle$ be proper. Then $S \cdot S^{*}+\mathbb{1}=S^{*}$.

Next, we give an explicit formula for the calculation of $S^{k}$. It can be proved by induction on $k$.

Lemma 3. If $S$ is a proper $n$-clock series, $k \in \mathbb{N}$, and $w \in\left(\mathbb{R}_{\geq 0}^{1+n}\right)\left(\Sigma \times \mathbb{R}_{\geq 0}^{1+n}\right)^{*}$, then $\left(S^{k}, w\right)$ has the explicit representation

$$
\left(S^{k}, w\right)=\sum_{w=w_{1} \cdot \ldots \cdot w_{k}} \prod_{i=1}^{k}\left(S, w_{i}\right)
$$

Rational Clock Series. For $c \in \mathcal{F}, k \in \mathcal{K}, \sigma \in \Sigma, \phi \in \Phi(X)$, and $\lambda \subseteq X$, we define the $\mathcal{F}$-monomial $\langle c, k, \sigma, \phi, \lambda\rangle:\left(\mathbb{R}_{\geq 0}^{1+n}\right)\left(\Sigma \times \mathbb{R}_{\geq 0}^{1+n}\right)^{*} \rightarrow K$ as follows:

$$
(\langle c, k, \sigma, \phi, \lambda\rangle, w)= \begin{cases}c(\delta) \odot k & \text { if } w=(t, \nu)\left(\sigma, t+\delta, \nu^{\prime}\right) \in\left(\mathbb{R}_{\geq 0}^{1+n}\right)\left(\Sigma \times \mathbb{R}_{\geq 0}^{1+n}\right) \\ & \text { s.t. } \delta \in \mathbb{R}_{\geq 0}, \nu+\delta \models \phi \text { and } \nu^{\prime}=\nu+\delta[\lambda:=0] \\ & \text { otherwise }\end{cases}
$$

An $n$-clock series $S$ is $\mathcal{F}$-rational if it can be defined starting from finitely many $\mathcal{F}$-monomials or the clock series $\mathbb{1}$ and $\mathbb{0}$, by means of a finite number of applications of,$+ \cdot$ and ${ }^{*}$, where the latter may only be applied to proper $n$ clock series. We use $\mathcal{K}_{n}^{\mathcal{F}-\text { rat }}\left\langle\left\langle\Sigma, \mathbb{R}_{\geq 0}\right\rangle\right\rangle$ to denote the set of all $\mathcal{F}$-rational $n$-clock series.

Observe that, similarly to the case of WTA, by restricting $\mathcal{K}$ and $\mathcal{F}$, we obtain rational expressions for several other (unweighted) automata classes or rational 
fps for weighted automata. For instance, if $\mathcal{K}$ is the Boolean semiring and $\mathcal{F}$ is the family of constant functions 1 , then rational clock series correspond to rational clock expressions defined by Bouyer and Petit [16].

Example 1. Consider the following specification of a real-time system with a single resource, where $\Lambda=\{a, b, c, d\}$ is a set of actions:

The system must execute $a$ and $b$, and $b$ must be executed exactly 3 time units after $a$. Between $a$ and $b$, action $c($ costs $€ 3)$ and action $d$ (costs $€ 2$ ) may be executed consecutively for an arbitrary number of times, but $d$ is restricted to happen strictly between 1 and 2 time units after $c$. Being in the state after action $a$ or $d$ has been executed, costs $€ 5$ per time unit, whereas being in the state after $c$ has been executed, costs $€ 1$ per time unit.

The specification can be represented by the following rational clock series over the tropical semiring, $\Lambda, Y=\{x, y\}$ and $C_{i}(\delta)=i \cdot \delta$ for each $i, \delta \in \mathbb{R}_{\geq 0}$ :

$$
\left\langle C_{0}, 0, a, \top,\{x\}\right\rangle\left(\left\langle C_{5}, 3, c, \top,\{y\}\right\rangle\left\langle C_{1}, 2, d, 1<y<2, \emptyset\right\rangle\right)^{*}\left\langle C_{5}, 0, b, x=3, \emptyset\right\rangle
$$

where $T$ means true. In Fig. 1, we give the corresponding WTA.

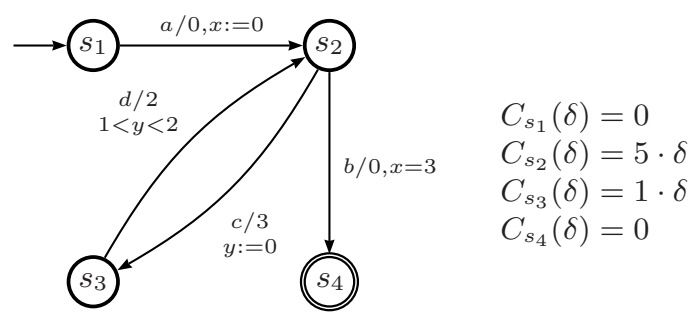

Fig. 1. The weighted timed automaton for Example 1

Recognizable Clock Series. We say that a clock series $S$ is an $\mathcal{F}$-recognizable $n$-clock series if there is a WTA $\mathcal{A}=\left(S, S_{0}, S_{f}, E, C\right)$ with $S=\|\mathcal{A}\|$. We use $\mathcal{K}_{n}^{\mathcal{F}-r e c}\left\langle\left\langle\Sigma, \mathbb{R}_{\geq 0}\right\rangle\right\rangle$ to denote the set of all $\mathcal{F}$-recognizable $n$-clock series.

Now, we are ready to present the main theorem of our paper.

Theorem 1. Let $\mathcal{K}$ be a semiring, $\Sigma$ be a finite alphabet, $X=\left\{x_{1}, \ldots, x_{n}\right\}$ be a finite set of clock variables and $\mathcal{F}$ be a family of functions from $\mathbb{R}_{\geq 0}$ to $K$. Then the set of $\mathcal{F}$-recognizable $n$-clock series is equal to the set of $\mathcal{F}$-rational $n$-clock series:

$$
\mathcal{K}_{n}^{\mathcal{F}-\text { rec }}\left\langle\left\langle\Sigma, \mathbb{R}_{\geq 0}\right\rangle\right\rangle=\mathcal{K}_{n}^{\mathcal{F}-r a t}\left\langle\left\langle\Sigma, \mathbb{R}_{\geq 0}\right\rangle\right\rangle
$$

Proof. The two inclusions of this result will follow from Proposition 1 and Proposition 2, respectively, presented in the next two sections. 


\section{Rationality Implies Recognizability}

In this section, we prove one inclusion of Theorem 1 namely that any rational $n$-clock series is recognizable. To this end, we will show that the basic $n$-clock series $\mathbb{1}, \mathbb{O}$ and $\mathcal{F}$-monomials are recognized by a WTA. Then, we will present new constructions that prove that WTA are closed under addition, Cauchy product and Kleene star iteration.

Proposition 1. $\mathcal{K}_{n}^{\mathcal{F}-r a t}\left\langle\left\langle\Sigma, \mathbb{R}_{\geq 0}\right\rangle\right\rangle \subseteq \mathcal{K}_{n}^{\mathcal{F}-r e c}\left\langle\left\langle\Sigma, \mathbb{R}_{\geq 0}\right\rangle\right\rangle$.

Proof. Follows from Lemmas 4 5, 7 and 9 given subsequently.

Lemma 4. $\mathbb{1}, \mathbb{O}$ and $\mathcal{F}$-monomials in $\mathcal{K}_{n}^{\mathcal{F}-\text { rat }}\left\langle\left\langle\Sigma, \mathbb{R}_{\geq 0}\right\rangle\right\rangle$ are recognizable $n$-clock series.

Proof. $\mathbb{0}$ is the behaviour of the WTA $\mathcal{A}_{\emptyset}=(\{s\},\{s\}, \emptyset, \emptyset, C)$, and the WTA $\mathcal{A}_{\mathbb{1}}=(\{s\},\{s\},\{s\}, \emptyset, C)$ corresponds to the clock series $\mathbb{1}$. In both cases, $C_{s} \in$ $\mathcal{F}$ is arbitrary. Let $S=\langle c, k, \sigma, \phi, \lambda\rangle$ be an $\mathcal{F}$-monomial in $\mathcal{K}_{n}^{\mathcal{F}-\text { rat }}\left\langle\left\langle\Sigma, \mathbb{R}_{\geq 0}\right\rangle\right\rangle$, where $c \in \mathcal{F}, k \in \mathcal{K}, \sigma \in \Sigma, \phi \in \Phi(X), \lambda \subseteq X$. We define the WTA $\mathcal{A}_{\langle c, k, \sigma, \phi, \lambda\rangle}=\left(S, S_{0}, S_{f}, E, C\right)$ where

$-S=\left\{s_{1}, s_{2}\right\}$

$-S_{0}=\left\{s_{1}\right\}$

$-S_{f}=\left\{s_{2}\right\}$

- $E=\left\{\left(s_{1}, s_{2}, \sigma, \phi, \lambda\right)\right\}$

$-C=\left\{C_{E}\right\} \cup\left\{C_{s}: s \in S\right\}$

where $C_{E}: E \rightarrow K$ is defined by $C_{E}\left(\left(s_{1}, s_{2}, \sigma, \phi, \lambda\right)\right)=k$. Also, let $C_{s_{1}}=c$, and choose any $C_{s_{2}} \in \mathcal{F}$. Clearly, $\left\|\mathcal{A}_{\langle c, k, \sigma, \phi, \lambda\rangle}\right\|=S$.

The proof of the next lemma can be done as in the case of traditional finite automata by taking a disjoint union of two WTA.

Lemma 5. If $S, T \in \mathcal{K}_{n}^{\mathcal{F}-r e c}\left\langle\left\langle\Sigma, \mathbb{R}_{\geq 0}\right\rangle\right\rangle$, then $S+T \in \mathcal{K}_{n}^{\mathcal{F}-r e c}\left\langle\left\langle\Sigma, \mathbb{R}_{\geq 0}\right\rangle\right\rangle$.

In the following, we give normalization techniques for WTA which will be essential for subsequent constructions of WTA. For showing closure of WTA under the Cauchy product, we need a final-location-normalization. We say that a WTA $\mathcal{A}$ is final-location-normalized if there is one single final location, and this location has no outgoing edge.

Lemma 6. If $\mathcal{A}$ is a WTA, then there is a final-location-normalized WTA $\mathcal{A}^{\prime}$ with $(\|\mathcal{A}\|, w)=\left(\left\|\mathcal{A}^{\prime}\right\|, w\right)$ for any $w \in\left(\mathbb{R}_{\geq 0}^{1+n}\right)\left(\Sigma \times \mathbb{R}_{\geq 0}^{1+n}\right)^{+}$.

Proof. Let $\mathcal{A}=\left(S, S_{0}, S_{f}, E, C\right)$ be a WTA.

Define $\mathcal{A}^{\prime}=\left(S^{\prime}, S_{0}^{\prime}, S_{f}^{\prime}, E \cup E^{\prime}, C \cup C^{\prime}\right)$, where

$-S^{\prime}=S \cup\left\{s_{f}\right\}$

$-S_{0}^{\prime}=S_{0}$

$-S_{f}^{\prime}=\left\{s_{f}\right\}$ 
- $E^{\prime}=\left\{\left(s, s_{f}, \sigma, \phi, \lambda\right): \exists s^{\prime} \in S_{f}\right.$ s.t. $\left.\left(s, s^{\prime}, \sigma, \phi, \lambda\right) \in E\right\}$

$-C^{\prime}: E^{\prime} \rightarrow K$ is defined by $C^{\prime}\left(\left(s, s_{f}, \sigma, \phi, \lambda\right)\right)=\sum_{\substack{s^{\prime} \in S_{f} \\\left(s, s^{\prime}, \phi, \phi\right.}} C\left(\left(s, s^{\prime}, \sigma, \phi, \lambda\right)\right)$

$-C_{s_{f}} \in \mathcal{F}$

Intuitively, we redirect all edges going into a final location to the new final location. The weight of each of these new edges must be the sum of the weights of all "equivalent" edges, i.e., edges with the same label, clock constraint and reset set. Using this notion of equivalence, we can show that $(\|\mathcal{A}\|, w)=\left(\left\|\mathcal{A}^{\prime}\right\|, w\right)$ for any $w \in\left(\mathbb{R}_{\geq 0}^{1+n}\right)\left(\Sigma \times \mathbb{R}_{\geq 0}^{1+n}\right)^{+}$.

Lemma 7. If $S, T \in \mathcal{K}_{n}^{\mathcal{F}-r e c}\left\langle\left\langle\Sigma, \mathbb{R}_{\geq 0}\right\rangle\right\rangle$, then $S \cdot T \in \mathcal{K}_{n}^{\mathcal{F}-r e c}\left\langle\left\langle\Sigma, \mathbb{R}_{\geq 0}\right\rangle\right\rangle$.

Proof. We give the construction of the WTA $\mathcal{A}$ such that $\|\mathcal{A}\|=S \cdot T$. For $i=1,2$, let $\mathcal{A}_{i}=\left(S^{i}, S_{0}^{i}, S_{f}^{i}, E^{i}, C^{i}\right)$ such that $\left\|\mathcal{A}_{1}\right\|=S$ and $\left\|\mathcal{A}_{2}\right\|=T$. By Lemma 6, we know that there is a final-location-normalized WTA $\mathcal{A}_{N}=\left(S^{N}, S_{0}^{N},\left\{s_{f}\right\}, E^{N}, C^{N}\right)$ such that $\left(\left\|\mathcal{A}_{1}\right\|, w\right)=\left(\left\|\mathcal{A}_{N}\right\|, w\right)$ for any $w \in\left(\mathbb{R}_{\geq 0}^{1+n}\right)\left(\Sigma \times \mathbb{R}_{\geq 0}^{1+n}\right)^{+}$. Assume $C^{N}=\left\{C_{E^{N}}^{N}\right\} \cup\left\{C_{s}^{N}\right\}_{s \in S^{N}}$ and $\left|S_{0}^{1} \cap S_{f}^{1}\right|=m$. Define $\mathcal{A}_{N, 2}=\left(S^{\bar{N}} \cup S^{2}, S_{0}^{N}, S_{f}^{2}, E^{N} \cup E^{2} \cup E,\left\{C^{N}\right\} \cup\left\{C^{2}\right\} \cup\left\{C_{E}\right\}\right)$ where

- $E=\left\{\left(s, s^{\prime}, \sigma, \phi, \lambda\right): s^{\prime} \in S_{0}^{2},\left(s, s_{f}, \sigma, \phi, \lambda\right) \in E^{N}\right\}$

$-C_{E}: E \rightarrow K$ is defined by $C_{E}\left(\left(s, s^{\prime}, \sigma, \phi, \lambda\right)\right)=C_{E^{N}}^{N}\left(\left(s, s_{f}, \sigma, \phi, \lambda\right)\right)$ if $\left(s, s_{f}, \sigma, \phi, \lambda\right) \in E^{N}$

Intuitively, we redirect all edges going into the single final location of $\mathcal{A}_{N}$ to the initial locations of $\mathcal{A}_{2}$ and preserve the cost assigned to these edges.

For $i \in \mathbb{N}$, define $\mathcal{A}_{2, i}$ to be an isomorphic copy of $\mathcal{A}_{2}$ such that its locations and edges are indexed by $i$. Let $\mathcal{A}$ be the disjoint union of $\mathcal{A}_{N, 2}$ and $\mathcal{A}_{2, i}$ for $1 \leq i \leq m$. Then, $(\|\mathcal{A}\|, w)=S \cdot T: \mathcal{A}_{N, 2}$ recognizes precisely the clock words $w$ that are the concatentation of two clock words $w_{1}$ and $w_{2}$ accepted by $\mathcal{A}_{N}$ and $\mathcal{A}_{2}$, respectively. Words obtained by concatentation of an empty word $w_{0} \in \Gamma_{n}$ and a word $w_{2}$ recognized by $\mathcal{A}_{1}$ and $\mathcal{A}_{2}$, respectively, have to be treated in a different manner. To overcome the problem that $\left(\left\|\mathcal{A}_{1}\right\|, w_{0}\right)=m \cdot 1$ (i.e., the sum of $m$ summands of $1 \in K)$, whereas $\left(\mathcal{A}_{N}, w_{0}\right)=0$ due to the construction of $\mathcal{A}_{N}$, we add $m$ isomorphic copies of $\mathcal{A}_{2}$. In this way, words of this kind are assigned the correct weight.

For showing closure of WTA under the Kleene star iteration, we need an additional normalization technique. A WTA is initial-location-normalized if all initial locations are sources, i.e., have no ingoing edges. Note that, in contrast to the case of classical weighted automata, we do not require to have one single initial state.

Lemma 8. If $\mathcal{A}$ is a WTA, then there is an initial-location-normalized WTA $\mathcal{A}^{\prime}$ with $(\|\mathcal{A}\|, w)=\left(\left\|\mathcal{A}^{\prime}\right\|, w\right)$ for any $w \in\left(\mathbb{R}_{\geq 0}^{1+n}\right)\left(\Sigma \times \mathbb{R}_{\geq 0}^{1+n}\right)^{*}$.

Proof. Let $\mathcal{A}=\left(S, S_{0}, S_{f}, E, C\right)$ be a WTA, where $C=\left\{C_{E}\right\} \cup\left\{C_{s}: s \in S\right\}$. For each $s \in S$, let $s^{\prime}$ be the copy of $s$.

Define $\mathcal{A}^{\prime}=\left(S \cup S^{\prime}, S_{0}^{\prime}, S_{f} \cup S_{f}^{\prime}, E \cup E^{\prime}, C \cup C^{\prime}\right)$, where 
$-S^{\prime}=\left\{s^{\prime}: s \in S_{0}\right\}$

$-S_{0}^{\prime}=S^{\prime}$

$-S_{f}^{\prime}=\left\{s^{\prime}: s \in S_{0} \cap S_{f}\right\}$

- $E^{\prime}=\left\{\left(s^{\prime}, t, \sigma, \phi, \lambda\right):(s, t, \sigma, \phi, \lambda) \in E\right\}$

$-C^{\prime}=\left\{C_{E^{\prime}}^{\prime}\right\} \cup\left\{C_{s^{\prime}}^{\prime}: s^{\prime} \in S^{\prime}\right\}$, where $C_{E^{\prime}}^{\prime}: E^{\prime} \rightarrow K$ is defined by $C_{E^{\prime}}^{\prime}\left(\left(s^{\prime}, t, \sigma, \phi, \lambda\right)\right)=C_{E}((s, t, \sigma, \phi, \lambda))$, and $C_{s^{\prime}}^{\prime}=C_{s}$ for any $s \in S$.

The intuituion behind this construction is to create a new initial location $s^{\prime}$ for every initial location $s \in S_{0}$ such that $s^{\prime}$ carries only copies of the outgoing edges of $s$. In particular, no (new) initial location has any ingoing edge. The final states of $\mathcal{A}^{\prime}$ consist of the final states of $\mathcal{A}$ and of those locations $s^{\prime}$ whose original location $s$ is both initial and final in $\mathcal{A}$. This choice of new final states $s^{\prime}$ guarantees that $\mathcal{A}^{\prime}$ behaves correctly on the empty n-clock words. One can prove $\left\|\mathcal{A}^{\prime}\right\|=\|\mathcal{A}\|$ by establishing a weight-preserving bijective correspondence between the successful runs of $\mathcal{A}$ and $\mathcal{A}^{\prime}$.

Corollary 1. Let $\mathcal{A}$ be a WTA. Then there is an initial- and final-locationnormalized WTA $\mathcal{A}_{N}$ with $\left(\left\|\mathcal{A}_{N}\right\|, w\right)=(\|\mathcal{A}\|, w)$ for any $w \in\left(\mathbb{R}_{\geq 0}^{1+n}\right)\left(\Sigma \times \mathbb{R}_{\geq 0}^{1+n}\right)^{+}$. Moreover, $\left(\left\|\mathcal{A}_{N}\right\|, w_{0}\right)=0$ for any $w_{0} \in \Gamma_{n}$.

Lemma 9. If $S \in \mathcal{K}_{n}^{\mathcal{F}-r e c}\left\langle\left\langle\Sigma, \mathbb{R}_{\geq 0}\right\rangle\right\rangle$ is proper, then $S^{*} \in \mathcal{K}_{n}^{\mathcal{F}-r e c}\left\langle\left\langle\Sigma, \mathbb{R}_{\geq 0}\right\rangle\right.$.

Proof. By Corollary 1 there is an initial- and final-location-normalized WTA $\mathcal{A}=\left(S, S_{0},\left\{s_{f}\right\}, E, C\right)$ with $\|\mathcal{A}\|=S$. We define

$\mathcal{A}^{*}=\left(S \cup\left\{s_{0 f}\right\}, S_{0} \cup\left\{s_{0 f}\right\},\left\{s_{f}, s_{0 f}\right\}, E \cup E^{\prime}, C \cup C^{\prime}\right)$, where

- $s_{0 f}$ is a new location (to obtain $\left(\left\|\mathcal{A}^{*}\right\|, w_{0}\right)=1$ for $\left.w_{0} \in \Gamma_{n}\right)$

- $E^{\prime}=\left\{\left(s, s^{\prime}, \sigma, \phi, \lambda\right): s^{\prime} \in S_{0},\left(s, s_{f}, \sigma, \phi, \lambda\right) \in E\right\}$

$-C^{\prime}: E^{\prime} \rightarrow K$ is defined by $C^{\prime}\left(\left(s, s^{\prime}, \sigma, \phi, \lambda\right)\right)=C_{E}\left(\left(s, s_{f}, \sigma, \phi, \lambda\right)\right)$ if $\left(s, s_{f}, \sigma, \phi, \lambda\right) \in E$

By a careful analysis of the successful runs of $\mathcal{A}^{*}$ and their weights, it can be shown that $\left\|\mathcal{A}^{*}\right\|=\|\mathcal{A}\|^{*}$, which implies the result.

\section{Recognizability Implies Rationality}

In this section, we show that any $n$-clock series recognized by a WTA $\mathcal{A}$ is rational by solving a system of equations induced by $\mathcal{A}$. The solution of the system corresponds to the rational clock series. Before we present the actual result, we give some lemmas. Let $\mathcal{A}=\left(S, S_{0}, S_{f}, E, C\right)$ be a WTA. For any two locations $s, s^{\prime} \in S$, we set $\mathcal{A}_{s, s^{\prime}}=\left(S,\{s\},\left\{s^{\prime}\right\}, E, C\right)$. The following lemma states how we can compute $\|\mathcal{A}\|$.

Lemma 10. If $\mathcal{A}=\left(S, S_{0}, S_{f}, E, C\right)$ is a WTA, then

$$
\|\mathcal{A}\|=\sum_{\left(s_{0}, s_{f}\right) \in S_{0} \times S_{f}}\left\|\mathcal{A}_{s_{0}, s_{f}}\right\|
$$


The next lemma shows that for the behaviour of any $\mathcal{A}_{s, s^{\prime}}$, we can give an equivalent linear equation. This can be proved by decomposing any successful run of $\mathcal{A}_{s, s^{\prime}}$ after the first discrete transition and replacing the first component by the corresponding monomial WTA, using laws of associativity and distributivity.

Lemma 11. Let $\mathcal{A}=\left(S, S_{0}, S_{f}, E, C\right)$ be a WTA, and assume that $s_{f} \in S_{f}$ is fixed. Then, for any $s \in S$,

$$
\left\|\mathcal{A}_{s, s_{f}}\right\|= \begin{cases}\sum_{\left(s, s^{\prime}, \sigma, \phi, \lambda\right) \in E}\left\|\mathcal{A}_{\left\langle C_{s}, k, \sigma, \phi, \lambda\right\rangle}\right\| \cdot\left\|\mathcal{A}_{s^{\prime}, s_{f}}\right\|+\mathbb{1} & \text { if } s=s_{f} \\ \sum_{\left(s, s^{\prime}, \sigma, \phi, \lambda\right) \in E}\left\|\mathcal{A}_{\left\langle C_{s}, k, \sigma, \phi, \lambda\right\rangle}\right\| \cdot\left\|\mathcal{A}_{s^{\prime}, s_{f}}\right\| & \text { otherwise }\end{cases}
$$

where $k=C_{E}\left(\left(s, s^{\prime}, \sigma, \phi, \lambda\right)\right)$.

The objective of these lemmas is to provide the basis for building a system of linear equations that represents the behaviour of a given WTA $\mathcal{A}$. The solution of this system correesponds to a rational clock series that is equivalent to the behaviour of $\mathcal{A}$. However, we need to show that it is guaranteed that there is such a solution. The next lemma supplies us with an even stronger result, namely that there is a unique solution.

Lemma 12. Let $S, S_{1}, S_{2} \in \mathcal{K}_{n}\left\langle\left\langle\Sigma, \mathbb{R}_{\geq 0}\right\rangle\right\rangle, S_{1}$ be proper. Then the equation $S=S_{1} \cdot S+S_{2}$ has the unique solution $T=S_{1}^{*} \cdot S_{2}$.

Finally, we present the crucial property between recognizable and rational clock series. For proving it, we use Lemmas [10, 11] and [12.

Proposition 2. $\mathcal{K}_{n}^{\mathcal{F}-r e c}\left\langle\left\langle\Sigma, \mathbb{R}_{\geq 0}\right\rangle\right\rangle \subseteq \mathcal{K}_{n}^{\mathcal{F}-r a t}\left\langle\left\langle\Sigma, \mathbb{R}_{\geq 0}\right\rangle\right.$.

\section{Timed Series}

As mentioned in Sect. 3, we use the clock semantics for defining a natural concatenation operation. However, research in the real-time community focuses on timed languages rather than clock languages. In this section, we show that a Kleene-Schützenberger theorem can be given for the corresponding class of fps, so-called timed series.

Timed Series. An fps $S:\left(\Sigma \times \mathbb{R}_{\geq 0}\right)^{*} \rightarrow K$ is called a timed series. We denote the set of timed series by $\mathcal{K}\left\langle\left\langle\left(\Sigma \times \mathbb{R}_{>0}\right)^{*}\right\rangle\right\rangle$. We say that a timed series $S \in \mathcal{K}\left\langle\left\langle\left(\Sigma \times \mathbb{R}_{\geq 0}\right)^{*}\right\rangle\right\rangle$ is recognizable if there is a WTA $\mathcal{A}$ such that $S=\|\mathcal{A}\|^{T}$. The set of recognizable timed series will be denoted by $\mathcal{K}^{r e c}\left\langle\left\langle\left(\Sigma \times \mathbb{R}_{\geq 0}\right)^{*}\right\rangle\right\rangle$.

Projection. The use of timed semantics rather than clock semantics sacrifices some significant information concerning the values of the clock variables that precludes us from defining the notion of rationality for timed series in the same way as for clock series. Therefore, we use the approach of Bouyer and 
Petit [16], and introduce a projection that maps clock series to timed series. Let $\pi:\left(\mathbb{R}_{>0}^{1+n}\right)\left(\Sigma \times \mathbb{R}_{>0}^{1+n}\right)^{*} \rightarrow\left(\Sigma \times \mathbb{R}_{\geq 0}\right)^{*}$ be the partial function defined by $\pi\left(\left(t_{0}, \nu_{0}\right)\left(\bar{\sigma}_{1}, t_{1}, \nu_{1}\right) \ldots\left(\bar{\sigma}_{k}, t_{k}, \nu_{k}\right)\right)=\left(\sigma_{1}, t_{1}\right) \ldots\left(\sigma_{k}, t_{k}\right)$ if $\left(t_{0}, \nu_{0}\right)=\left(0,0^{n}\right)$, undefined otherwise. We extend $\pi$ to a function $\bar{\pi}: \mathcal{K}_{n}^{r e c}\left\langle\left\langle\Sigma, \mathbb{R}_{\geq 0}\right\rangle\right\rangle \rightarrow \mathcal{K}^{r e c}\langle\langle(\Sigma \times$ $\left.\left.\left.\mathbb{R}_{\geq 0}\right)^{*}\right\rangle\right\rangle: S \mapsto \bar{\pi}(S)$ where

$$
\left(\bar{\pi}(S), w_{T}\right)=\sum_{\substack{w_{C} \in\left(\mathbb{R}_{\geq 0}^{1+n}\right)\left(\Sigma \times \mathbb{R}_{\geq 0}^{1+n}\right)^{*} \\ \pi\left(w_{C}\right)=w_{T}}}\left(S, w_{C}\right)
$$

for any timed word $w_{T} \in\left(\Sigma \times \mathbb{R}_{\geq 0}\right)^{*}$. Notice that the sum in the equation is finite: for any recognizable timed word, there is only a finite number of $n$-clock words $w_{C}$ in $\pi^{-1}\left(w_{T}\right)$ such that $\left(\|\mathcal{A}\|, w_{C}\right) \neq 0$, because there is only a finite number of runs on any clock word $w_{C}$.

Rational Timed Series. A timed series $S \in \mathcal{K}\left\langle\left\langle\left(\Sigma \times \mathbb{R}_{\geq 0}\right)^{*}\right\rangle\right\rangle$ is rational if it can be defined by a single application of $\bar{\pi}$ to a rational $n$-clock series $T \in$ $\mathcal{K}_{n}^{r a t}\left\langle\left\langle\Sigma, \mathbb{R}_{\geq 0}\right\rangle\right\rangle$, i.e., $S=\bar{\pi}(T)$. We use $\mathcal{K}^{r a t}\left\langle\left\langle\left(\Sigma \times \mathbb{R}_{\geq 0}\right)^{*}\right\rangle\right\rangle$ to mean the set of rational timed series.

The following lemma gives the relation between recognizable timed series and recognizable clock series.

Lemma 13. Let $\mathcal{A}$ be a WTA and $w_{T} \in\left(\Sigma \times \mathbb{R}_{\geq 0}\right)^{*}$ be a timed word. Then $\left(\|\mathcal{A}\|^{T}, w_{T}\right)=\left(\bar{\pi}\left(\|\mathcal{A}\|^{C}\right), w_{T}\right)$.

Corollary 2. $\mathcal{K}^{\text {rat }}\left\langle\left\langle\left(\Sigma \times \mathbb{R}_{\geq 0}\right)^{*}\right\rangle\right\rangle=\mathcal{K}^{r e c}\left\langle\left\langle\left(\Sigma \times \mathbb{R}_{\geq 0}\right)^{*}\right\rangle\right\rangle$.

Proof. The definition of rational timed series and Lemma 13 ensure that both rational and recognizable timed series correspond to a single application of $\bar{\pi}$ to a rational (recognizable, respectively) $n$-clock series. This and Theorem 1 imply the result.

\section{Conclusion}

We have presented a new definition of WTA for modelling consumption of resources, and we have obtained a Kleene-Schützenberger theorem. Our definition over a semiring is more general than definitions given in the literature so far and emphasizes the relation to weighted automata. The Kleene-Schützenberger theorem for WTA provides an alternative characterization of the possible behaviours of WTA. The crucial point for obtaining this result was to find new normalization techniques that allow for the construction of Cauchy product- and Kleene star-WTA. We point out that due to the cost functions assigned to the locations it is not possible to use standard normalization techniques for weighted automata.

Apart from being a fundamental theoretical result, Kleene's theorem is also of practical interest. Kleene's theorem for the set of regular languages is used for automata-based verification purposes: the rational expression is considered 
to be the specification of the system, which, by the Kleene theorem, can be transformed into an equivalent finite automaton. It is a fascinating challenge to investigate whether our result can be used in the same manner.

In our paper, we have shown that WTA are closed under addition, Cauchy product and Kleene star iteration. Moreover, the corresponding constructions are effective. It is of great practical interest whether we get similar positive results for other standard properties and decidability problems. In particular, we want to investigate the emptiness problem, i.e., given a WTA $\mathcal{A}$, whether $\|\mathcal{A}\|=\mathbb{0}$. There is a good reason to hope for a positive result, as both the emptiness problem of timed automata and weighted automata is decidable, where the latter result applies to weighted automata where the semiring is a field.

Another interesting direction for future work is to consider whether there is a Büchi-type theorem for WTA, i.e., are weighted timed automata expressively equivalent to some weighted timed version of monadic second-order logic. This should combine methods of Wilke 31 and Droste and Gastin 19. The present closure results for rational operations provide a promising starting point.

\section{References}

1. Alur, R., Bernadsky, M., Madhusudan, P.: Optimal reachability in weighted timed games. In: Díaz, J., Karhumäki, J., Lepistö, A., Sannella, D. (eds.) ICALP 2004. LNCS, vol. 3142, pp. 122-133. Springer, Heidelberg (2004)

2. Alur, R., Dill, D.L.: A theory of timed automata. Theoretical Computer Science 126(2), 183-235 (1994)

3. Alur, R., La Torre, S., Pappas, G.J.: Optimal paths in weighted timed automata. In: Di Benedetto, M.D., Sangiovanni-Vincentelli, A.L. (eds.) HSCC 2001. LNCS, vol. 2034, pp. 49-62. Springer, Heidelberg (2001)

4. Alur, R., Madhusudan, P.: Decision problems for timed automata: A survey. In: Bernardo, M., Corradini, F. (eds.) SFM-RT 2004. LNCS, vol. 3185, pp. 1-24. Springer, Heidelberg (2004)

5. Asarin, E., Caspi, P., Maler, O.: A Kleene theorem for timed automata. In: LICS 1997, pp. 160-171. IEEE Computer Society Press, Los Alamitos (1997)

6. Asarin, E., Caspi, P., Maler, O.: Timed regular expressions. Journal of the ACM 49(2), 172-206 (2002)

7. Asarin, E., Dima, C.: Balanced timed regular expressions. In: Vogler, W., Larsen, K.G. (eds.) MTCS. ENTCS, vol. 68, pp. 16-33. Elsevier, Amsterdam (2002)

8. Behrmann, G., Fehnker, A., Hune, T., Larsen, K., Pettersson, P., Romijn, J., Vaandrager, F.: Minimum-cost reachability for priced timed automata. In: Di Benedetto, M.D., Sangiovanni-Vincentelli, A.L. (eds.) HSCC 2001. LNCS, vol. 2034, pp. 147161. Springer, Heidelberg (2001)

9. Di Benedetto, M.D., Sangiovanni-Vincentelli, A.L. (eds.): HSCC 2001. LNCS, vol. 2034. Springer, Heidelberg (2001)

10. Berstel, J., Reutenauer, C.: Rational Series and their Languages. Springer, New York, USA (1988)

11. Bouyer, P., Brihaye, T., Bruyère, V., Raskin, J.-F.: On the optimal reachability problem on weighted timed automata. Formal Methods in System Design 31(2), 135-175 (2007) 
12. Bouyer, P., Brihaye, T., Markey, N.: Improved undecidability results on weighted timed automata. Inf. Process. Lett. 98(5), 188-194 (2006)

13. Bouyer, P., Brinksma, E., Larsen, K.G.: Optimal infinite scheduling for multi-priced timed automata. Formal Methods in System Design (to appear, 2007)

14. Bouyer, P., Larsen, K.G., Markey, N.: Model-checking one-clock priced timed automata. In: Seidl, H. (ed.) FOSSACS 2007. LNCS, vol. 4423, pp. 108-122. Springer, Heidelberg (2007)

15. Bouyer, P., Petit, A.: Decomposition and composition of timed automata. In: Wiedermann, J., Van Emde Boas, P., Nielsen, M. (eds.) ICALP 1999. LNCS, vol. 1644, pp. 210-219. Springer, Heidelberg (1999)

16. Bouyer, P., Petit, A.: A Kleene/Büchi-like theorem for clock languages. J. Autom. Lang. Comb. 7(2), 167-186 (2001)

17. Bozga, M., Daws, C., Maler, O., Olivero, A., Tripakis, S., Yovine, S.: Kronos: A model-checking tool for real-time systems. In: Hu, A.J., Vardi, M.Y. (eds.) CAV 1998. LNCS, vol. 1427, pp. 546-550. Springer, Heidelberg (1998)

18. Brihaye, T., Bruyère, V., Raskin, J.-F.: Model-checking weighted timed automata. In: Lakhnech, Y., Yovine, S. (eds.) FORMATS 2004 and FTRTFT 2004. LNCS, vol. 3253, pp. 277-292. Springer, Heidelberg (2004)

19. Droste, M., Gastin, P.: Weighted automata and weighted logics. Theor. Comput. Sci. 380(1-2), 69-86 (2007)

20. Fox, M., Long, D.: Modelling mixed discrete-continuous domains for planning. Journal of AI Research 27, 235-297 (2006)

21. Henzinger, T.: The theory of hybrid automata. In: LICS 1996, pp. 278-292. IEEE Computer Society Press, Los Alamitos (1996)

22. Henzinger, T.A., Ho, P.-H., Wong-Toi, H.: HYTECH: A model checker for hybrid systems. International Journal on Software Tools for Technology Transfer 1(1-2), 110-122 (1997)

23. Kristoffersen, K., Larsen, K., Pettersson, P., Weise, C.: VHS Case Study 1 - Experimental Batch Plant using UPPAAL, BRICS, University of Aalborg, Denmark (May 1999)

24. Kuich, W., Salomaa, A.: Semirings, Automata, Languages. EATCS Monographs on Theoretical Computer Science, vol. 5. Springer, Berlin (1986)

25. Larsen, K.G., Pettersson, P., Yi, W.: UPPAAL in a nutshell. International Journal on Software Tools for Technology Transfer 1(1-2), 134-152 (1997)

26. Larsen, K.G., Rasmussen, J.I.: Optimal conditional reachability for multi-priced timed automata. In: Sassone, V. (ed.) FOSSACS 2005. LNCS, vol. 3441, pp. 234 249. Springer, Heidelberg (2005)

27. Niebert, P., Yovine, S.: Computing optimal operation schemes for chemical plants in multi-batch mode. In: Lynch, N.A., Krogh, B.H. (eds.) HSCC 2000. LNCS, vol. 1790, Springer, Heidelberg (2000)

28. Illum Rasmussen, J., Larsen, K.G., Subramani, K.: Resource-optimal scheduling using priced timed automata. In: Jensen, K., Podelski, A. (eds.) TACAS 2004. LNCS, vol. 2988, pp. 220-235. Springer, Heidelberg (2004)

29. Salomaa, A., Soittola, M.: Automata-Theoretic Aspects of Formal Power Series. Springer, New York (1978)

30. Schützenberger, M.P.: On the definition of a family of automata. Information and Control 4, 245-270 (1961)

31. Wilke, T.: Specifying Timed State Sequences in Powerful Decidable Logics and Timed Automata. In: Langmaack, H., de Roever, W.-P., Vytopil, J. (eds.) FTRTFT 1994 and ProCoS 1994. LNCS, vol. 863, pp. 694-715. Springer, Heidelberg (1994) 